\title{
Effects of Phenyllactic Acid as Sanitizing Agent for Inactivation of
}

\section{Listeria monocytogenes Biofilms}

Fang Liu ${ }^{\text {a\# }}$, Lihui Du ${ }^{\text {b\# }}$, Tong Zhao ${ }^{c^{*}}$, Ping Zhao ${ }^{c}$, Michael P. Doyle ${ }^{c}$

${ }^{\text {a } I n s t i t u t e ~ o f ~ A g r i c u l t u r a l ~ P r o d u c t s ~ P r o c e s s i n g, ~ J i a n g s u ~ A c a d e m y ~ o f ~ A g r i c u l t u r a l ~ S c i e n c e s, ~}$ Nanjing 210014, China

${ }^{\mathrm{b}}$ College of Food Science and Engineering, Collaborative Innovation Center for Modern

Grain Circulation and Safety, Key Laboratory of Grains and Oils Quality Control and

Processing, Nanjing University of Finance and Economics, Nanjing 210023, China

${ }^{\mathrm{c}}$ Center for Food Safety, College of Agriculture and Environmental Sciences, University of Georgia, 1109 Experiment Street, Griffin, GA 30223, USA

\#Fang Liu and Lihui Du contributed equal to this project

*Corresponding author, tongzhao@uga.edu 


\begin{abstract}
The efficacy of phenyllactic acid (PLA) to inactivate Listeria monocytogenes planktonic cells and biofilms was determined and compared with the killing effects of lactic acid (LA), and levulinic acid (LVA) with sodium dodecyl sulfate (SDS). L. monocytogenes biofilms of different maturities, i.e., $37{ }^{\circ} \mathrm{C}$ for 3 and $7 \mathrm{~d}$ and $15{ }^{\circ} \mathrm{C}$ for 4 and $7 \mathrm{~d}$, were produced on 24-well flat-bottom polystyrene plates and treated with the sanitizers, including 3\% PLA, $1 \%$ PLA, $0.5 \%$ PLA, $0.25 \%$ PLA, 3\% LA, 1\% LA, and 3\% LVA plus 2\% SDS for 5, 10, 30, 60 min, respectively. The results of pure culture assays revealed that $1 \%$ PLA reduced the population of L. monocytogenes by $7 \log \mathrm{CFU} / \mathrm{ml}$ within $1 \mathrm{~min}$. The biofilms assays revealed that L. monocytogenes biofilms can be inactivated to different degrees by the sanitizer treatments. The killing effect of the sanitizers was increased as exposure time and sanitizer concentrations were increased. The sanitizers of 3\% PLA, 1\% PLA, and 3\% LVA plus $2 \%$ SDS effectively inactivated the early mature biofilm after a 5-min treatment, whereas $3 \%$ PLA was better than all the other sanitizers, including 1\% PLA, 3\% LA, 3\% LVA and 2\% SDS for inactivation of the late mature biofilm after a 5-min treatment. Confocal laser scanning microscopy analysis revealed that bacterial cell damages in the biofilm were enhanced as the PLA concentrations and exposure times were increased. These results suggested that PLA was effective in inactivating L. monocytogenes and its biofilm, even for the late mature biofilm.
\end{abstract}

KEYWORDS: Listeria monocytogenes, biofilm, phenyllactic acid, lactic acid 


\section{Introduction}

Listeria monocytogenes is an important foodborne pathogen that is able to persist in food processing environments (Farber \& Peterkin, 1991; Gandhi \& Chikindas, 2007), and can attach to form a biofilm to a variety of surfaces commonly present in a food processing facility (Alonso, Perry, Regeimbal, Regan, \& Higgins, 2014; Beresford, Andrew, \& Shama, 2001; Gamble \& Muriana, 2007; Zhao et al., 2013). Biofilms are defined as matrix-enclosed microorganisms that adhere to a surface and/or to each other, producing a dynamic environment in which the component microbial cells may reach homeostasis, and may optimally organize to make use of all available nutrients (Costerton, Lewandowski, Caldwell, Korber, \& Lappin-Scott, 1995). The presence of Listeria biofilms in food processing plants is an important hazard because the biofilm can contaminate food products that contact or pass over contaminated surfaces which can lead to an outbreak of listeriosis. Several studies have revealed that $L$. monocytogenes cells in biofilms are difficult to eliminate and can exhibit resistance to chlorine, acid anionic, and quaternary ammonium sanitizers (Frank \& Koffi, 1990; Martínez-Suárez, Ortiz, \& López-Alonso, 2016). Considering these issues, it is important to develop novel, safe and effective sanitizers to inactivate L. monocytogenes biofilms.

3-Phenyllactic acid (2-hydroxy-3-phenylpropanoic acid or $\beta$-phenyllactic acid, PLA), an organic acid, has been reported as an antimicrobial compound with broad-spectrum activity against bacteria, including L. monocytogenes (Dieuleveux, Van Der Pyl, Chataud, \& Gueguen, 1998). Dieuleveux et al. (1998) initially purified and identified the novel anti-Listeria compound, PLA, which is produced and excreted by Geotrichum candidum. Subsequently, it was determined that PLA is produced by a wide range of lactic acid bacteria species, such as Lactobacillus, Enterococcus, Weissella, and Leuconostoc, and has broad antibacterial activity that can inhibit a wide range of Gram-positive bacteria, including Staphylococcus aureus, Enterococcus faecalis, and Bacillus cereus, and Gram-negative bacteria, including Salmonella 
enterica and Escherichia coli (Mu, Yu, Zhu, Zhang, \& Jiang, 2012). In addition to its antibacterial activity, PLA can also inhibit yeasts and fungi (Lavermicocca, Valerio, \& Visconti, 2003; Lavermicocca, Valerio, Evidente, Lazzaroni, Corsetti, \& Gobbetti, 2000; Schwenninger et al., 2008). Hence, PLA may be a natural antibacterial substance that can control pathogens in contaminated food products and processing plants.

Although it has been reported that PLA can inhibit L. monocytogenes growth in culture medium and milk (Dieuleveux \& Gueguen, 1998), there are few reports that address its activity against L. monocytogenes in biofilms. The L. monocytogenes strains isolated from food and patients were previously typed to four major serotypes $(1 / 2 \mathrm{a}, 1 / 2 \mathrm{~b}, 1 / 2 \mathrm{c}$, and $4 \mathrm{~b})$ (Doumith, Buchrieser, Glaser, Jacquet, \& Martin, 2004). Although L. monocytogenes serotype $4 \mathrm{~b}$ is considered the most virulent, serotype $1 / 2 \mathrm{c}$ is the strongest biofilm former among the most frequently occurring serotypes in food and food processing environments (Norwood \& Gilmour, 1999; Lundén, Miettinen, Autio, \& Korkeala, 2000, Harvey, Keenan, \& Gilmour, 2007). Hence, Listeria monocytogenes ATCC 5779 (serotype 1/2c, cheese isolate) was used in this study. The objective of this study was to evaluate the effectiveness of PLA to inactivate $L$. monocytogenes in biofilms of different maturities.

\section{Materials and methods}

\subsection{Bacterial strain and preparation of a single-species culture}

Listeria monocytogenes ATCC 5779 (serotype 1/2c, cheese isolate) was stored at $-30{ }^{\circ} \mathrm{C}$. Before each experiment, the culture was thawed and first grown at $37{ }^{\circ} \mathrm{C}$ in Brain Heart Infusion broth (BHI, Becton Dickinson, Sparks, MD) for $24 \mathrm{~h}$, and then streaked on BHI agar and grown at $37{ }^{\circ} \mathrm{C}$ for $24 \mathrm{~h}$. A single bacterial colony was inoculated into a test tube containing $10 \mathrm{ml}$ of $\mathrm{BHI}$ medium, and transferred at least three times at $24-\mathrm{h}$ intervals before use. The final bacteria were grown to the exponential phase to obtain a bacterial count of about $10^{9} \mathrm{CFU} / \mathrm{ml}$, with the number of listeria confirmed by plating serial dilutions (1:10) on BHI agar. 


\subsection{Preparation of sanitizers}

The sanitizers, including PLA, LA, and LVA and SDS (Sigma, St. Louis, MO) were diluted in deionized water to the concentrations (w/v) to be evaluated in each experiment. The $\mathrm{pH}$ of the sanitizer solutions is shown in Table 1.

\subsection{Sanitizer sensitivity assay}

The protocol of the Association of Official Analytical Chemists was used to determine the sensitivity of L. monocytogenes ATCC 5779 to PLA and other sanitizers as described in a previous report (Zhao, Doyle, Zhao, Blake, \& Wu, 2001). L. monocytogenes ATCC 5779 suspension $(1 \mathrm{ml})$ was added to $199 \mathrm{~mL}$ of sanitizer solution (1\% PLA, 0.5\% PLA, $0.25 \%$ PLA, 3\% LA, $1 \%$ LA, $1 \%$ LA+ 0.5\% PLA, 1\% LA+ 0.25\% PLA, 0.5\% LA+ 0.25\% PLA, 0.5\% LA $+0.5 \%$ PLA, $3 \%$ LVA+ $2 \%$ SDS) being stirred with a magnetic stir bar at $100 \mathrm{rpm}$ in a 500-ml Erlenmeyer flask. At predetermined sampling times, $1.0 \mathrm{ml}$ of the treated bacterial suspension was removed and mixed with $9.0 \mathrm{ml}$ of $0.1 \mathrm{M}$ phosphate buffer (PBS) to neutralize the $\mathrm{pH}$. Then, the solution was serially (1:10) diluted in $0.1 \%$ peptone water, and $0.1 \mathrm{ml}$ of each dilution was surface-plated on BHI agar plates in duplicate. The plates were held at $37{ }^{\circ} \mathrm{C}$ for $48 \mathrm{~h}$ and L. monocytogenes colonies were enumerated. If no Listeria colonies were present by the direct plating method, a selective enrichment was applied. A 1.0-ml portion of bacterial suspension from each tube was added to a test tube containing $9 \mathrm{ml}$ of BHI broth and the tubes were incubated at $37{ }^{\circ} \mathrm{C}$ for $24 \mathrm{~h}$. A loopful of broth was surface streaked on each of duplicate MOX agar plates that were then held at $37{ }^{\circ} \mathrm{C}$ for $24 \mathrm{~h}$, and up to 5 colonies typical of L. monocytogenes (black) were confirmed by biochemical and immunological methods as we described previously (Zhao, Doyle, \& Zhao, 2004). All experiments were duplicated, and averages of results are reported

\subsection{Biofilm formation and sanitizer treatment}

Approximately $10^{7} \mathrm{CFU}(1 \mathrm{ml})$ of L. monocytogenes were added into each well of 24-well flat-bottom polystyrene plates (Costar, Corning, NY). The plates were incubated 
statically at $37{ }^{\circ} \mathrm{C}$ for $3 \mathrm{~d}$ and at $15{ }^{\circ} \mathrm{C}$ for $4 \mathrm{~d}$ or at $37{ }^{\circ} \mathrm{C}$ and at $15{ }^{\circ} \mathrm{C}$ for $7 \mathrm{~d}$. To maintain bacterial viability, BHI broth was changed every $24 \mathrm{~h}$ by aspirating old medium along the walls of each well, the well was rinsed three times with $0.1 \%$ peptone to remove the unbounded cells, and fresh medium was then added into each well. After incubation, the supernatant fluid was removed, and the wells were rinsed three times with $2 \mathrm{ml}$ of $0.01 \mathrm{M}$ phosphate buffer saline (PBS, Sigma, St. Louis, MO). The plates were then air dried for 5 min at $21{ }^{\circ} \mathrm{C}$. Four concentrations of PLA (3\% PLA, 1\% PLA, $0.5 \%$ PLA, $0.25 \%$ PLA) and two concentrations of LA (3\% LA, 1\% LA) were used in this study as sanitizer treatments. Sterile water was the negative control and the sanitizer of 3\% LVA and 2\% SDS was used as positive control. The water or chemicals were removed by aspiration after the 5, 10, 30, or 60-min treatment. After sanitizer treatment, the wells were filled with $2 \mathrm{ml}$ of N/E neutralizing broth (Becton Dickinson) for $10 \mathrm{~min}$ before aspiration, and then, $1 \mathrm{ml}$ of PBS was added into each well. The content of each well was harvested by scraping the surface thoroughly with a sterile polyester-tipped swab (15.2 cm, Fisher Scientific, Pittsburgh, PA). The PBS containing released biofilm cells and the swab were added to $9 \mathrm{ml}$ of $0.01 \mathrm{M} \mathrm{PBS,} \mathrm{pH} \mathrm{7.25,} \mathrm{and}$ vigorously agitated by a Vortex (G-560, Scientific Industries, Bohemia, NY) at $250 \mathrm{rpm}$ for 1 min. Cell suspension was serially $(1: 10)$ diluted and a volume of $0.1 \mathrm{ml}$ from each diluted tube were spread-plated in duplicate onto BHI plates. The plates were incubated at $37^{\circ} \mathrm{C}$ for $48 \mathrm{~h}$ for bacterial enumeration. The level of inactivation was expressed as the $\log _{10}$ reduction in the cell survival ratio for the chemical treatments $\left(\log \mathrm{N} / \mathrm{N}_{0}\right)$. $\mathrm{N}$ refers to the bacterial counts after chemical treatments, whereas $\mathrm{N}_{0}$ refers to the bacterial counts following water incubation (negative control biofilms).

\subsection{Confocal laser scanning microscopy (CLSM)}

For CLSM, a 1\% diluted exponential phase culture of L. monocytogenes ATCC 5779 was

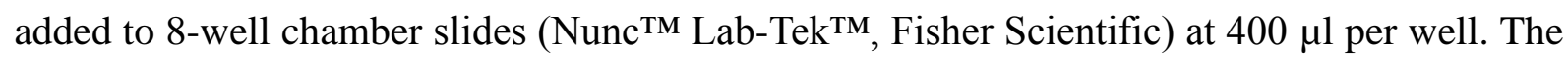
plates were incubated at $37^{\circ} \mathrm{C}$ for $3 \mathrm{~d}$ before exposure to sanitizer treatments. The medium 
was changed every $24 \mathrm{~h}$ to maintain bacterial viability. Prior to the treatment step, the wells were washed with $800 \mu \mathrm{l}$ of $0.01 \mathrm{M}$ PBS and air-dried for $10 \mathrm{~min}$, and after treatments they were filled with N/E neutralizing broth to neutralize the residual sanitizer solutions. To visualize cells, fluorescent dyes were used by applying the LIVE/DEAD BacLight ${ }^{\mathrm{TM}}$ viability kit (Molecular Probes, Life Technologies, Eugene, OR). The staining mixture was prepared by adding $3 \mu \mathrm{l}$ of component $\mathrm{A}$ and $3 \mu \mathrm{l}$ of component $\mathrm{B}$ per $1 \mathrm{~mL}$ of sterile saline. Freshly prepared staining mixture $(400 \mu \mathrm{l})$ was added to each well and incubated at $21^{\circ} \mathrm{C}$ for $15 \mathrm{~min}$ in the dark. After incubation, the wells were washed with PBS as described above. The wells were further filled with $2.5 \%$ glutaraldehyde in $0.1 \mathrm{M}$ cacodylate buffer (Sigma) and incubated for $30 \mathrm{~min}$ at room temperature to fix the specimen. The slides were removed from the chambers and then covered with coverslips with the use of BacLight ${ }^{\mathrm{TM}}$ Mounting Oil (Molecular Probes, Life Technologies, Eugene, OR). The samples were analyzed with a Zeiss LSM700 CLSM (Carl Zeiss Microscopy, Thornwood, NY) using Plan-Apochromat 63 oil-immersion, numerical aperture 1.4.

\subsection{Statistical analysis}

The sensitivity of L. monocytogenes ATCC 5779 biofilms to PLA and the other sanitizers was determined by three independent trials with duplicate plating $(n=6)$. Data were analyzed for analysis of variance (ANOVA) by SPSS software (version19.0, IBM-SPSS Inc., Armonk, NY) to determine least significant differences $(p<0.05)$ among the treatments. Principal component analysis (PCA) was conducted using the Unscrambler 9.7 software (Camo, USA) to discriminate the influence of different factors: culture temperature, growth time, exposure time and sanitizers on biofilm inactivation.

\section{Results}

\subsection{Bacterial inactivation assay}

The results of the inactivation of L. monocytogenes ATCC 5779 planktonic bacterial by PLA, LA and the other sanitizers are shown in Table 1 . The 3\% LVA plus $2 \%$ SDS and $1 \%$ 
PLA treatments inactivated L. monocytogenes ATCC 5779 to undetectable levels within 1 min, whereas $3 \%$ LA required at least 20 min to inactivate the same cell populations of listeria. PLA of $0.5 \%$ and $0.25 \%$ inactivated approximately $10^{7} \mathrm{CFU} / \mathrm{ml}$ of L. monocytogenes in 30 min and $60 \mathrm{~min}$, respectively, whereas viable strains were still detected by enrichment culture after treatment with $1 \%$ LA for 180 min. Interestingly, both the combined treatment of $1 \%$ LA $+0.5 \%$ PLA, and $0.5 \% \mathrm{LA}+0.5 \%$ PLA inactivated about $10^{7} \mathrm{CFU} / \mathrm{ml}$ of L. monocytogenes in 20 min, which is more effective than the treatment of 3\% LA only. The combined treatment of $1 \% \mathrm{LA}+0.25 \% \mathrm{PLA}$, or $0.5 \% \mathrm{LA}+0.25 \% \mathrm{PLA}$, inactivated approximately $10^{7} \mathrm{CFU} / \mathrm{ml}$ of L. monocytogenes in $30 \mathrm{~min}$, and $60 \mathrm{~min}$, respectively.

\subsection{Inactivation of L. monocytogenes in Biofilms}

The effectiveness of PLA, lactic acid (LA) and the other sanitizers including levulinic acid (LVA) and sodium dodecyl sulfate (SDS) on the inactivation of L. monocytogenes cells in biofilms grown to different maturities is revealed in Fig. 1 and Fig. 2, respectively. The results revealed that the listeria in the biofilms could not be completely inactivated by all of the sanitizers even when treated for $60 \mathrm{~min}$.

The effectiveness of the sanitizers on the inactivation of listeria in biofilms grown at $37^{\circ} \mathrm{C}$ for $3 \mathrm{~d}$ is revealed in Fig. 1A. Significant $(\mathrm{P}<0.05)$ reductions of 4.06, 3.94 and $3.32 \mathrm{log}$ CFU/ml were observed for 3\% PLA, 1\% PLA and 3\% LVA + 2\% SDS treatments, respectively, compared with the other sanitizers, including 3\% LA after a 5-min treatment (Fig. 1A). As the treatment time was increased, the effectiveness of $0.5 \%$ PLA, $0.25 \%$ PLA, 3\% LA and $1 \%$ LA significantly increased $(\mathrm{P}<0.05)$ (Fig. 1A). Treatment with $0.5 \%$ PLA for 30 and $60 \mathrm{~min}$, reduced listeria populations by 3.99 and $5.10 \mathrm{log}$, respectively, for which there was no significant difference $(\mathrm{P}>0.05)$ for treatments of 3\% PLA, 1\% PLA, 3\% LA, and 3\% LVA and $2 \%$ SDS.

The effectiveness of the sanitizers on the inactivation of listeria cells in biofilms grown at $37^{\circ} \mathrm{C}$ for $7 \mathrm{~d}$ is revealed in Fig. 1B. The initial Listeria counts in these biofilms were 
approximately $10 \log \mathrm{CFU} / \mathrm{ml}$, indicating that these biofilms were more densely populated with listeria than those treated in the studies described above. The trends of the inactivation of listeria populations in these late mature biofilms were similar. 3\% PLA reduced 3.40 log CFU $(\mathrm{P}<0.05) / \mathrm{ml}$ in the biofilm after a 5-min treatment, which was a significantly greater reduction of listeria than treatment with 1\% PLA, 0.5\% PLA, 0.25\% PLA, 3\% LA, 1\% LA, and 3\% LVA $+2 \%$ SDS which reduced 0.92, 0.33, 0.20, 0.88, 0.28, and $2.04 \log$ CFU/ml bacterial cells, respectively (Fig. 1B). Increasing the treatment time, significantly increased $(\mathrm{P}<0.05)$ the effectiveness of $1 \%$ PLA, or 3\% LVA and 2\% SDS (Fig. 1B). A 30- or 60-min treatment with $3 \%$ PLA, $1 \%$ PLA or $3 \%$ LVA plus $2 \%$ SDS were not significantly different in inactivating listeria in the late mature biofilms, and these treatments were significantly more effective than the other sanitizers, including 3\% LA and 0.5\% PLA (Fig. 1B).

Inactivation trends of listeria in biofilms grown at $15^{\circ} \mathrm{C}$ for $4 \mathrm{~d}$ and $7 \mathrm{~d}$ are revealed in Fig. 2 and are similar to the results shown in Fig. 1. 3\% PLA, 1\% PLA, and 3\% LVA + 2\% SDS were significantly $(\mathrm{P}<0.05)$ more effective with reductions of $4.51,4.07$ and $4.19 \log \mathrm{CFU} / \mathrm{ml}$, respectively, after 5 min treatment (Fig. 2A), compared to $0.5 \%$ PLA, $0.25 \%$ PLA, 3\% LA and $1 \%$ LA which reduced the listeria populations by $1.39,0.24,2.07$ and $0.36 \log \mathrm{CFU} / \mathrm{ml}$, respectively (Fig. 2A). The effectiveness of PLA, LA and the other sanitizer combinations on the inactivation of listeria in biofilms grown at $15^{\circ} \mathrm{C}$ for $7 \mathrm{~d}$ is revealed in Fig. 2B. 3\% PLA reduced $3.51 \log \mathrm{CFU}$ of listeria/ml after a 5-min treatment, which was significantly $(\mathrm{P}<0.05)$ more effective than the other sanitizers of 1\% PLA, 0.5\% PLA, 0.25\% PLA, 3\% LA, 1\% LA, and $3 \%$ LVA plus $2 \%$ SDS which reduced $1.09,0.48,0.35,0.94,0.36$, and $2.48 \log$ CFU of listeria/ml, respectively (Fig. 2B).

The effectiveness of the combined treatment of $1 \% \mathrm{LA}+0.5 \%$ PLA, and $1 \% \mathrm{LA}+0.25 \%$ PLA on the inactivation of listeria biofilms was also studied. $1 \%$ LA $+0.5 \%$ PLA had the same effectiveness on inactivating listeria in biofilms as that of 3\% LA for all the treatment exposure times (Fig. 1 and Fig. 2). The effectiveness of the sanitizers of 1\% LA + 0.5\% PLA, 
and $1 \%$ LA $+0.25 \%$ PLA significantly increased with increased treatment times (Fig. 1 and Fig. 2).

\subsection{Biofilm formation characteristics as determined by CLSM analysis}

CLSM images revealed that L. monocytogenes was able to form three-dimensional structures during the incubation time that was used. The L. monocytogenes biofilm formed on the chamber was uneven. The biofilm located near the center of the chamber was thinner, with an average height of ca. $2 \mu \mathrm{m}$ after $3 \mathrm{~d}$ at $37^{\circ} \mathrm{C}$ than that located along the walls of the chamber that had an average height of ca. $6 \mu \mathrm{m}$. The biofilm formed at the corners of the chamber was the thickest, at up to $8 \sim 10 \mu \mathrm{m}$.

Disintegration of the bacterial cell membrane of the listeria in the biofilm of ca. $6 \mu \mathrm{m}$ following treatment with the sanitizers for $10 \mathrm{~min}$ and $60 \mathrm{~min}$ was revealed in Fig. 3. The red brightness of the CLSM images was enhanced when the treatment concentrations of PLA and treatment time were increased, revealing that more bacterial cells in the biofilm were damaged, which was in concordance with the bacterial counts of viable cells in the biofilms after being treated with PLA. Although the layers of the biofilm treated with 1\% PLA, 3\% PLA and 3\% LVA and 2\% SDS for 60 min appeared almost entirely red, several locations within the biofilm were green indicating that some bacterial cells were still intact and viable.

\section{Discussion}

Many studies revealed that there are different stages in biofilm development (de la Fuente-Núñez, Reffuveille, Fernández, \& Hancock, 2013; Monds \& O'Toole, 2009; Thuptimdang P, Limpiyakorn, McEvoy, Prüß, \& Khan, 2015). There are at least four stages in biofilm formation: planktonic, attachment, maturation, and dispersion. According to the features of biofilm development, mature biofilms are significantly more resistant to sanitizers and they can disperse planktonic bacterial cells to the environment. Hence, four different $L$. monocytogenes biofilms of different maturities that were grown at different temperatures and times were used to evaluate the effectiveness of the sanitizers. A principal component analysis 
(PCA) was to determine if the maturity of the biofilm and sanitizer exposure time influenced biofilm inactivation of listeria (Fig. 4A). The PCA diagram of three clusters revealed that culture time and sanitizer exposure time can affect biofilm inactivation (Fig. 4A). Samples for Cluster 3 were all treated with the sanitizer for up to 30 and $60 \mathrm{~min}$, regardless of the maturity of the biofilm These results revealed that given enough exposure time the chemicals could penetrate the exopolysaccharide (EPS) barrier to inactivate the bacterial cells in the biofilm. Samples for Cluster 1 were of biofilms grown at $37^{\circ} \mathrm{C}$ for $3 \mathrm{~d}$ and $15^{\circ} \mathrm{C}$ for $4 \mathrm{~d}$ that were treated with sanitizers for $5 \mathrm{~min}$, and samples for Cluster 2 were of biofilms grown at $37^{\circ} \mathrm{C}$ and $15^{\circ} \mathrm{C}$ for $7 \mathrm{~d}$ that were treated with sanitizers for $5 \mathrm{~min}$ and $10 \mathrm{~min}$, respectively. These results revealed that the bacterial cells in these biofilms were more difficult to inactivate as biofilm maturity increased. The increased resistance of biofilms with increased maturity may be due to the thickness of the matrix of glycoproteins, EPS and other compounds (glycocalyx) surrounding biofilm cells which can act to protect cells physically from surface-active agents (Uhlich, Cooke, \& Solomon, 2006). Similar conclusions were reported in previous studies. Lee \& Frank (1991) reported that listeria cells in biofilms grown on stainless steel surfaces for 8 days were twice as resistant to hypochlorite than the cells grown for 4 days and 100 times more resistant as cells that were grown for only 4 h. Yang, Kendall, Medeiros, \& Sofos (2009) determined that the nine sanitizers they evaluated were more effective for reducing bacterial cells in 7-day-old biofilms grown on smooth surface high-density polyethylene coupons than the cells in 21-day-old biofilms. Beauchamp et al. (2012) determined that E. coli O157:H7 cells in biofilms became less sensitive to most sanitizer treatments as biofilm age increased.

Levulinic acid (LVA) with sodium dodecyl sulfate (SDS) has been reported previously to be an effective sanitizer for inactivating foodborne pathogens (Magnone, Marek, Sulakvelidze, \& Senecal, 2013; Zhao, Zhao, \& Cannon, Doyle, 2011; Zhao, Zhao, \& Doyle, 2009). Chen, Zhao, \& Doyle (2015) reported that different concentrations of LVA plus SDS (0.5\% LVA + $0.05 \%$ SDS, $1 \% \mathrm{LVA}+0.1 \% \mathrm{SDS}$, and $3 \% \mathrm{LVA}+2 \% \mathrm{SDS}$ ) were all more effective against 
the three pathogens in biofilms grown on stainless steel compared to the other commonly used sanitizers, including a commercial quaternary ammonium-based sanitizer (150 ppm), lactic acid (3\%), sodium hypochlorite (100 ppm), and hydrogen peroxide (2\%). Hence, $3 \%$ LVA and $2 \% \operatorname{SDS}$ was used in this study as the positive control to compare against the effectiveness of PLA. The effectiveness of the sanitizers to biofilms of different maturities was comprehensively analyzed for different treatment times by PCA methods (Fig. 5B, 5C, 5D). Fig. 4B classified the sanitizers into three different clusters based on the bacterial reduction to the samples of early mature biofilm (3-d old biofilm at $37{ }^{\circ} \mathrm{C}$ and 4 -d old biofilm at $15^{\circ} \mathrm{C}$ ) treated for $5 \mathrm{~min}$ and $10 \mathrm{~min}$. The PCA diagram was segregated into three clusters. Cluster 3 included the sanitizers, i.e., 3\% PLA, 1\% PLA, and 3\% LVA and 2\% SDS, indicating that these three sanitizers were similar in their effectiveness to inactivate the biofilms of early maturity with a short sanitizer exposure time. Cluster 2 included the sanitizers $3 \%$ LA, $0.5 \%$ PLA, $1 \%$ LA and $0.5 \%$ PLA, and $1 \%$ LA, and $0.25 \%$ PLA. Based on listeria reduction in late mature biofilms (7-d old biofilm at $37^{\circ} \mathrm{C}$ and $15^{\circ} \mathrm{C}$ ) treated for 5 min and $10 \mathrm{~min}$, the sanitizers were distributed into two different clusters and two different points (Fig. 4C). Results revealed that the effectiveness of the sanitizers to eliminate listeria in late mature biofilms occurred in the following order: 3\% PLA, 3\% LVA and 2\% SDS, and then the sanitizers discussed in Clusters 1 and 2. The sanitizers were distributed in Fig. 4D into two different clusters based on the listeria reduction in all the biofilm samples treated for $30 \mathrm{~min}$ and 60 min. Cluster 1 included the sanitizers of 3\% PLA, 3\% LVA+2\% SDS, $1 \%$ PLA, 3\% LA, $0.5 \%$ PLA, $1 \%$ LA+0.5\% PLA, and $1 \%$ LA+0.25\% PLA, indicating that all of the above sanitizers had similar efficacy in reducing listeria with long exposure times. The sanitizers in low concentrations can gradually inactivate listeria as the exposure time was increased. de Oliveiraa, Brugneraa, Cardosob, Alvesc, \& Piccolia (2010) reported that dead cells inactivated by the sanitizers can protect other Listeria cells in the biofilm, and this may help explain why the effectiveness of high concentrations of sanitizers did not increase with 
increased exposure times. Based on the above results, we can conclude that PLA was most effective in reducing listeria on biofilms compared with the other sanitizers evaluated, and may be a useful sanitizer in food processing facilities. A combination of $1 \%$ LA plus $0.5 \%$ PLA provides the same degree of effectiveness of 3\% LA, which may enable the use of lower amounts of PLA in sanitizer preparations.

Biofilm development of listeria is influenced by the material characteristics of the matrix to which the listeria attached as well as surface roughness of this material. (Teughels, Van Assche, Sliepen, \& Quirynen, 2006). Chen, Zhao, \& Doyle (2015) reported that exposure to 3\% LVA plus $2 \%$ SDS for $10 \mathrm{~min}$ can reduce listeria populations by ca. $8 \log \mathrm{CFU} / \mathrm{ml}$. In this study, $3 \%$ LVA $+2 \%$ SDS for 10 min only reduced listeria populations by ca. 4 log reduction. The difference for this $\log$ reduction may be attributed to the different materials on which the biofilms were grown. Since stainless steel that was used only had a flat surface structure, the biofilms that were formed along the walls of the 24-well flat-bottom polystyrene plates were thicker than those grown on the stainless steel, hence it was more difficult for the chemicals to penetrate the EPS barrier to inactivate the listeria cells in the biofilm. Gomes, Moreira, Simões, Melo, \& Mergulhão (2014) reported that biofilms were mainly formed on the walls and not on the bottom of the wells in 96-well microtiter plates. In our study, it was also determined by CLSM analysis that the biofilms grown along the corners of each well was much thicker than those grown on the bottom. This may help explain why the biofilms existing in the crevices of equipment in food processing facilities are more difficult to eliminate than those grown on equipment surfaces. These results suggest that equipment design could be improved by eliminating sharp angle connections where biofilms are more likely to form.

In conclusion, PLA was effective in inactivating both L. monocytogenes planktonic cells and listeria cells in biofilms, and 3\% PLA was more effective than 3\% LA, or 3\% LVA plus 2\% SDS in reducing listeria populations in more resistant biofilms of older maturity. 


\section{Acknowledgments}

This study was funded by the National Key Technology Support Program of China (2015BAD16B04) and the National Natural Science Foundation of China (31371802). The laboratory reagents and supplies were in part funded by Agriculture and Food Research Initiative Grant No. 2011-68003-30012 from the USDA National Institute of Food and Agriculture. Fang Liu thanks the Jiangsu Government Scholarship program for supporting her studies at the University of Georgia.

\section{References}

Alonso, A. N., Perry, K. J., Regeimbal, J. M., Regan, P. M., \& Higgins, D. E. (2014). Identification of Listeria monocytogenes determinants required for biofilm formation. PLoS One, 9, e113696.

Beauchamp, C. S., Dourou, D., Geornaras, I., Yoon, Y., Scanga, J. A, Belk, K. E., et al. (2012). Sanitizer efficacy against Escherichia coli O157:H7 biofilms on inadequately cleaned meat-contact surface materials. Food Protection Trends, 32, 173-182.

Beresford, M. R., Andrew, P. W., \& Shama, G. (2001). Listeria monocytogenes adheres to many materials found in food-processing environments. Journal of Applied Microbiology, 90, $1000-1005$.

Chen, D., Zhao, T., \& Doyle, M. P. (2015). Control of pathogens in biofilms on the surface of stainless steel by levulinic acid plus sodium dodecyl sulfate. International Journal of Food Microbiology, 207, 1-7.

Costerton, J. W., Lewandowski, Z., Caldwell, D. E., Korber, D. R., \& Lappin-Scott, H. M. (1995). Microbial biofilms. Annual Review of Microbiology, 49, 711-745.

de la Fuente-Núñez, C., Reffuveille, F., Fernández, L., \& Hancock, R. E. (2013). Bacterial biofilm development as a multicellular adaptation: antibiotic resistance and new 
therapeutic strategies. Current Opinion in Microbiology, 16, 580-589.

de Oliveiraa, M. M. M., Brugneraa, D. F., Cardosob, M. G., Alvesc, E., \& Piccolia, R. H. (2010). Disinfectant action of Cymbopogon sp. essential oils in different phases of biofilm formation by Listeria monocytogenes on stainless steel surface. Food Control, 21, 549-553.

Dieuleveux, V., \& Gueguen, M. (1998). Antimicrobial effects of D-3-phenyllactic acid on Listeria monocytogenes in TSB-YE medium, milk, and cheese. Journal of Food Protection, $61,1281-1285$

Dieuleveux, V., Van Der Pyl, D., Chataud, J., \& Gueguen, M. (1998) Purification and characterization of anti-Listeria compounds produced by Geotrichum candidum. Applied and Environmental Microbiology, 64, 800-803.

Doumith, M., Buchrieser, C., Glaser, P., Jacquet, C., \& Martin, P. (2004). Differentiation of the major Listeria monocytogenes serovars by multiplex PCR. Journal of Clinical Microbiology, 42, 3819-3822.

Farber, J. M., \& Peterkin, P. I. (1991) Listeria monocytogenes, a food-borne pathogen. Microbiology Reviews, 55, 476-511.

Frank, J. F., \& Koffi, R. A. Surface-adherent growth of Listeria monocytogenes is associated with increased resistance to surfactant sanitizers and heat. Journal of Food Protection, 53, 550-554(5)

Gamble, R., \& Muriana, P. M. (2007). Microplate fluorescence assay for measurement of the ability of strains of Listeria monocytogenes from meat and meat processing plants to adhere to abiotic surfaces. Applied and Environmental Microbiology, 73, 5235-5244.

Gandhi, M., \& Chikindas, M. L. (2007) Listeria: A foodborne pathogen that knows how to survive. International Journal of Food Microbiology, 113, 1-15.

Gomes, L. C., Moreira, J. M. R., Simões, M., Melo, L. F., \& Mergulhão, F. J. (2014). Biofilm localization in the vertical wall of shaking 96-well plates. Scientifica, 2014, 231083.

Harvey, J., Keenan, K. P., \& Gilmour, A. (2007). Assessing biofilm formation by Listeria 
monocytogenes strains. Food Microbiology, 24, 380-392.

Lavermicocca, P., Valerio, F., Evidente, A., Lazzaroni, S., Corsetti, A., \& Gobbetti, M. (2000). Purification and characterization of novel antifungal compounds from the sourdough Lactobacillus plantarum strain 21B. Applied and Environmental Microbiology, 66, 4084-4090.

Lavermicocca, P., Valerio, F., \& Visconti, A. (2003). Antifungal activity of phenyllactic acid against molds isolated from bakery products. Applied and Environmental Microbiology, $69,634-640$

Lee, S., \& Frank, J. F. (1991). Inactivation of surface-adherent Listeria monocytogenes using hypochlorite and heat. Journal of Food Protection, 54, 4-6.

Lundén, J. M., Miettinen, M. K., Autio, T. J., \& Korkeala, H. J. (2000). Persistent Listeria monocytogenes strains show enhanced adherence to food contact surface after short contact times. Journal of Food Protection, 63, 1204-1207.

Magnone, J.P., Marek, P.J., Sulakvelidze, A., \& Senecal, A.G. (2013). Additive approach for inactivation of Escherichia coli O157:H7, Salmonella, and Shigella spp. on contaminated fresh fruits and vegetables using bacteriophage cocktail and produce wash. Journal of Food Protection, 76, 1336-1341.

Martínez-Suárez, J. V., Ortiz, S., \& López-Alonso, V. (2016). Potential impact of the resistance to quaternary ammonium disinfectants on the persistence of Listeria monocytogenes in food processing environments. Front Microbiology, 7, 638.

Monds, R. D., \& O'Toole, G. A. (2009). The developmental model of microbial biofilms: ten years of a paradigm up for review. Trends in Microbiology, 17, 73-87.

Mu, W., Yu, S., Zhu, L., Zhang, T., \& Jiang, B. (2012). Recent research on 3-phenyllactic acid, a broad-spectrum antimicrobial compound. Applied and Environmental Microbiology, $95,1155-1163$.

Norwood, D. E., \& Gilmour, A. (19990. Adherence of Listeria monocytogenes strains to 
stainless steel coupons. Journal of Applied Microbiology, 86, 576-582.

Schwenninger, S. M., Lacroix, C., Truttmann, S., Jans, C., Sporndli, C., Bigler, L., et al. (2008). Characterization of low-molecular-weight antiyeast metabolites produced by a food-protective Lactobacillus-Propionibacterium coculture. Journal of Food Protection, 71, $2481-2487$.

Teughels, W., Van Assche, N., Sliepen, I., \& Quirynen, M. (2006). Effect of material characteristics and/or surface topography on biofilm development. Clinical Oral Implants Research, s2, 68-81.

Thuptimdang, P., Limpiyakorn, T., McEvoy, J., Prüß, B. M., \& Khan, E. (2015). Effect of silver nanoparticles on Pseudomonas putida biofilms at different stages of maturity. Journal of Hazardous Materials, 290, 127-133.

Uhlich, G. A., Cooke, P. H., \& Solomon, E. B. (2006). Analyses of the red-dry-rough phenotype of an Escherichia coli O157:H7 strain and its role in biofilm formation and resistance to antibacterial agents. Applied and Environmental Microbiology, 72, 2564-2572.

Yang, H., Kendall, P. A., Medeiros, L. C., \& Sofos, J. N. (2009). Efficacy of sanitizing agents against Listeria monocytogenes biofilms on high-density polyethylene cutting board surfaces. Journal of Food Protection, 72, 990-998.

Zhao, T., Doyle, M. P., \& Zhao, P. (2004). Control of Listeria monocytogenes in a biofilm by competitive-exclusion microorganisms. Applied and Environmental Microbiology, 70, $3996-4003$.

Zhao, T., Doyle, M. P., Zhao, P., Blake, P., \& Wu, F. M. (2001). Chlorine inactivation of Escherichia coli O157:H7 in water. Journal of Food Protection, 64, 1607-1609.

Zhao, T., Podtburg, T. C., Zhao, P., Chen, D., Baker, D. A., Cords, B., et al. (2013). Reduction by competitive bacteria of Listeria monocytogenes in biofilms and Listeria bacteria in floor drains in a ready-to-eat poultry processing plant. Journal of Food Protection, 76, $601-607$. 
Zhao, T., Zhao, P., \& Doyle, M. P. (2009). Inactivation of Salmonella and Escherichia coli $\mathrm{O} 157: \mathrm{H} 7$ on lettuce and poultry skin by combinations of levulinic acid and sodium dodecyl sulfate. Journal of Food Protection, 72, 928-936.

Zhao, T., Zhao, P., Cannon, J. L., \& Doyle, M. P. (2011). Inactivation of Salmonella in biofilms and on chicken cages and preharvest poultry by levulinic acid and sodium dodecyl sulfate. Journal of Food Protection, 74, 2024-2230. 
TABLE 1. Inactivation of $L$. monocytogenes ATCC 5779 planktonic cells by different sanitizers at $21 \pm 2{ }^{\circ} \mathrm{C}$

\begin{tabular}{|c|c|c|c|c|c|c|c|c|c|c|}
\hline \multirow{2}{*}{ Sanitizers (pH) } & \multicolumn{10}{|c|}{ L. monocytogenes ATCC $5779\left(\log _{10} \mathrm{CFU} / \mathrm{mL}\right)$ at $\mathrm{min}:$} \\
\hline & 0 & 1 & 2 & 5 & 10 & 20 & 30 & 60 & 120 & 180 \\
\hline $\mathrm{H}_{2} \mathrm{O}$ & $7.07 \pm 0.11$ & $6.93 \pm 0.03$ & $7.02 \pm 0.07$ & $7.01 \pm 0.07$ & $6.96 \pm 0.06$ & $7.01 \pm 0.04$ & $7.02 \pm 0.02$ & $7.10 \pm 0.01$ & $6.99 \pm 0.12$ & $6.97 \pm 0.13$ \\
\hline $1 \% \operatorname{PLA}(2.72)$ & $6.90 \pm 0.02$ & $a_{-}$ & - & - & - & - & - & - & - & - \\
\hline $0.5 \%$ PLA (2.89) & $6.93 \pm 0.02$ & $6.88 \pm 0.02$ & $6.39 \pm 0.08$ & $5.52 \pm 0.02$ & $4.77 \pm 0.02$ & $2.34 \pm 0.12$ & - & - & - & - \\
\hline $0.25 \%$ PLA (2.95) & $7.06 \pm 0.07$ & $7.06 \pm 0.09$ & $6.99 \pm 0.04$ & $6.96 \pm 0.07$ & $6.95 \pm 0.05$ & $6.96 \pm 0.16$ & $6.88 \pm 0.04$ & $6.65 \pm 0.01$ & - & - \\
\hline $3 \%$ LA (2.35) & $5.59 \pm 0.09$ & $4.78 \pm 0.02$ & $4.54 \pm 0.06$ & $3.89 \pm 0.03$ & $3.41 \pm 0.18$ & - & - & - & - & - \\
\hline $1 \%$ LA (2.56) & $6.89 \pm 0.10$ & $6.72 \pm 0.08$ & $6.79 \pm 0.02$ & $6.61 \pm 0.02$ & $6.43 \pm 0.02$ & $6.15 \pm 0.05$ & $6.08 \pm 0.02$ & $5.6 \pm 0.06$ & ${ }^{\mathrm{b}}<1.7$ & $<1.7$ \\
\hline $1 \% \mathrm{LA}+0.5 \% \mathrm{PLA}(2.53)$ & $5.42 \pm 0.19$ & $4.62 \pm 0.10$ & $4.31 \pm 0.11$ & $3.39 \pm 0.02$ & $<1.7$ & - & - & - & - & - \\
\hline $1 \% \mathrm{LA}+0.25 \% \mathrm{PLA}(2.53)$ & $6.43 \pm 0.04$ & $6.05 \pm 0.04$ & $5.79 \pm 0.02$ & $4.56 \pm 0.04$ & $3.21 \pm 0.09$ & $<1.7$ & - & - & - & - \\
\hline $0.5 \% \mathrm{LA}+0.5 \% \mathrm{PLA}(2.63)$ & $6.64 \pm 0.05$ & $6.01 \pm 0.04$ & $5.10 \pm 0.06$ & $3.99 \pm 0.01$ & $<1.7$ & - & - & - & - & - \\
\hline $\begin{array}{c}0.5 \% \mathrm{LA}+0.25 \% \mathrm{PLA} \\
(2.67)\end{array}$ & $6.84 \pm 0.01$ & $6.52 \pm 0.01$ & $6.35 \pm 0.01$ & $5.93 \pm 0.03$ & $5.09 \pm 0.06$ & $3.15 \pm 0.11$ & $<1.7$ & - & - & - \\
\hline $3 \% \mathrm{LVA}+2 \% \mathrm{SDS}(2.83)$ & - & - & - & - & - & - & - & - & - & - \\
\hline
\end{tabular}

${ }^{-}$, not detectable by direct plating and an enrichment procedure.

$3 \quad{ }^{b}<1.7$, detectable by an enrichment procedure but not by direct plating.

4 PLA = 3-Phenyllactic acid, LA = lactic acid, LVA = levulinic acid, SDS = sodium dodecyl sulfate. 
1 Fig. 1. Inactivation of L. monocytogenes ATCC 5779 in biofilms grown at $37^{\circ} \mathrm{C}$ for $3 \mathrm{~d}(\mathrm{~A})$ and $7 \mathrm{~d}$ (B) after treatment with different 2 concentrations of PLA for 5, 10, 30, and $60 \mathrm{~min}$. The bars represent mean values \pm standard deviations (n=6). A different capital letter at each 3 point indicates a significant difference between different treatments for the same treatment time $(\mathrm{P}<0.05)$. A different lower case letter at each 4 point indicates a significant difference between the same treatment for a different treatment time $(\mathrm{P}<0.05)$. PLA = 3-Phenyllactic acid, LA = 5 lactic acid, $\mathrm{LVA}=$ levulinic acid, SDS = sodium dodecyl sulfate.

6 Fig. 2. Inactivation of $L$. monocytogenes ATCC 5779 in biofilms grown at $15^{\circ} \mathrm{C}$ for $4 \mathrm{~d}$ (A) and $7 \mathrm{~d}$ (B) after treatment with different 7 concentrations of PLA and other sanitizers for 5, 10, 30, and $60 \mathrm{~min}$. The bars represent mean values \pm standard deviations ( $\mathrm{n}=6$ ). A different 8 capital letter at each point indicates a significant difference between different treatments for the same treatment time $(\mathrm{P}<0.05)$. A different lower 9 case letter at each point indicates a significant difference between the same treatment for a different treatment time $(\mathrm{P}<0.05)$. PLA $=$ 10 3-Phenyllactic acid, LA = lactic acid, LVA = levulinic acid, SDS = sodium dodecyl sulfate.

11 Fig. 3. Representative CLSM images of L. monocytogenes ATCC 5779 biofilms. The biofilms were grown in $\mathrm{BHI}$ at $37^{\circ} \mathrm{C}$ for 3 days $(\mathrm{A}-1$ and 12 A-2), and treated for 5 min with 0.5\% PLA (B-1), 1\% PLA (C-1), 3\% PLA (D-1), 3\%LVA+2\%SDS (E-1), and 60 min with $0.5 \%$ PLA (B-2), 1\% 13 PLA (C-2), $3 \%$ PLA (D-2), 3\%LVA+2\%SDS (E-2). The scale bar is $10 \mu \mathrm{m}$. PLA = 3-Phenyllactic acid, LA = lactic acid, LVA = levulinic acid, 14 SDS = sodium dodecyl sulfate.

15 Fig. 4. Principal component analysis (PCA) diagrams revealing the discrimination of mean score factors for listeria inactivation in biofilms 
which evaluates the influence of growth temperatures $\left(37^{\circ} \mathrm{C} / 15^{\circ} \mathrm{C}\right)$, growth time $(3 \mathrm{~d} / 4 \mathrm{~d} / 7 \mathrm{~d})$ and sanitizer exposure times $(5 / 10 / 30 / 60 \mathrm{~min})(\mathrm{A})$,

17 and the influence of different sanitizers on (B), early mature biofilms treated for $5 \mathrm{~min}$ and $10 \mathrm{~min}$; (C), late mature biofilms treated for 5 min

18 and $10 \mathrm{~min}$; (D), all biofilm samples treated for $30 \mathrm{~min}$ and $60 \mathrm{~min}$;) on L. monocytogenes ATCC 5779 biofilms. PLA = 3-Phenyllactic acid, LA

19 = lactic acid, LVA = levulinic acid, SDS = sodium dodecyl sulfate. 

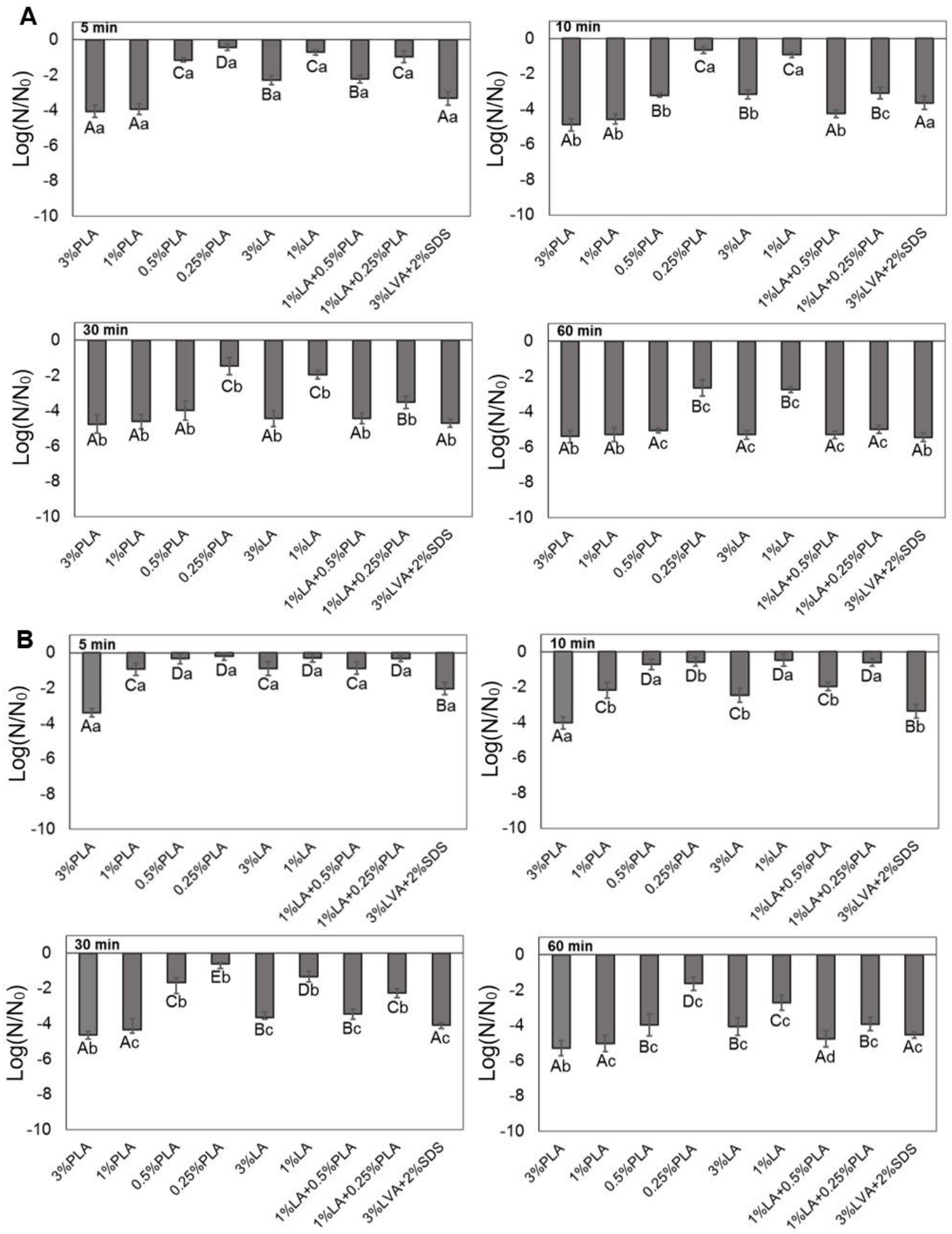

Fig. 1. 

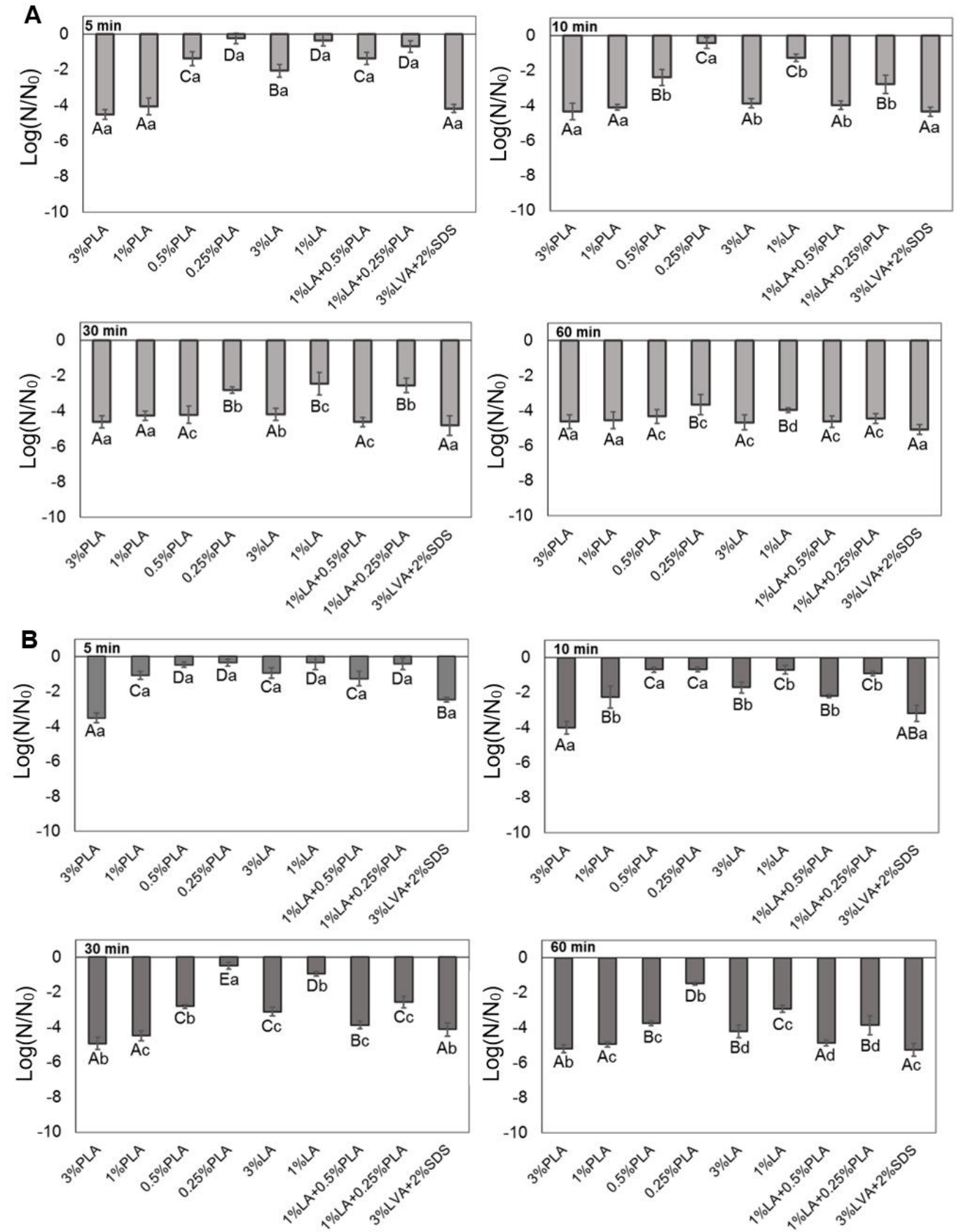

\author{
Fig. 2.
}



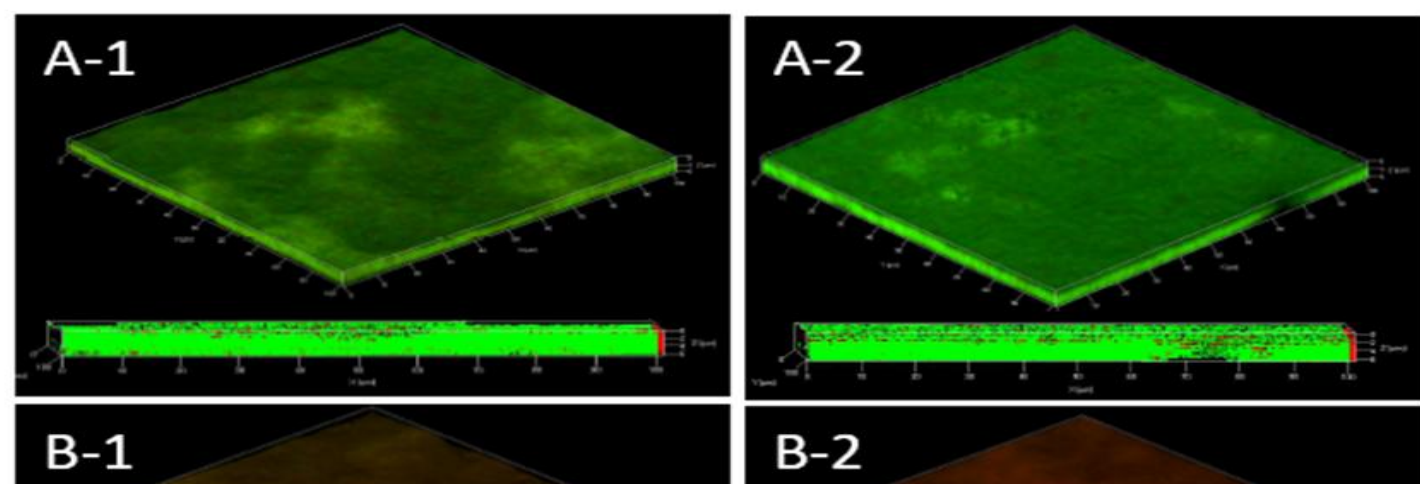

B-1

B-2

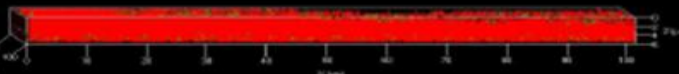

C-1
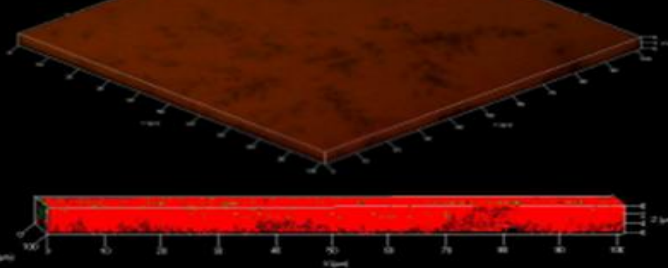

D-1

D-2

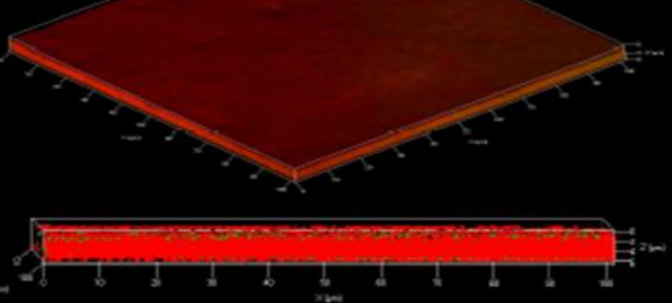

$$
\text { E-1 }
$$$$
\text { E-2 }
$$

Fig. 3. 

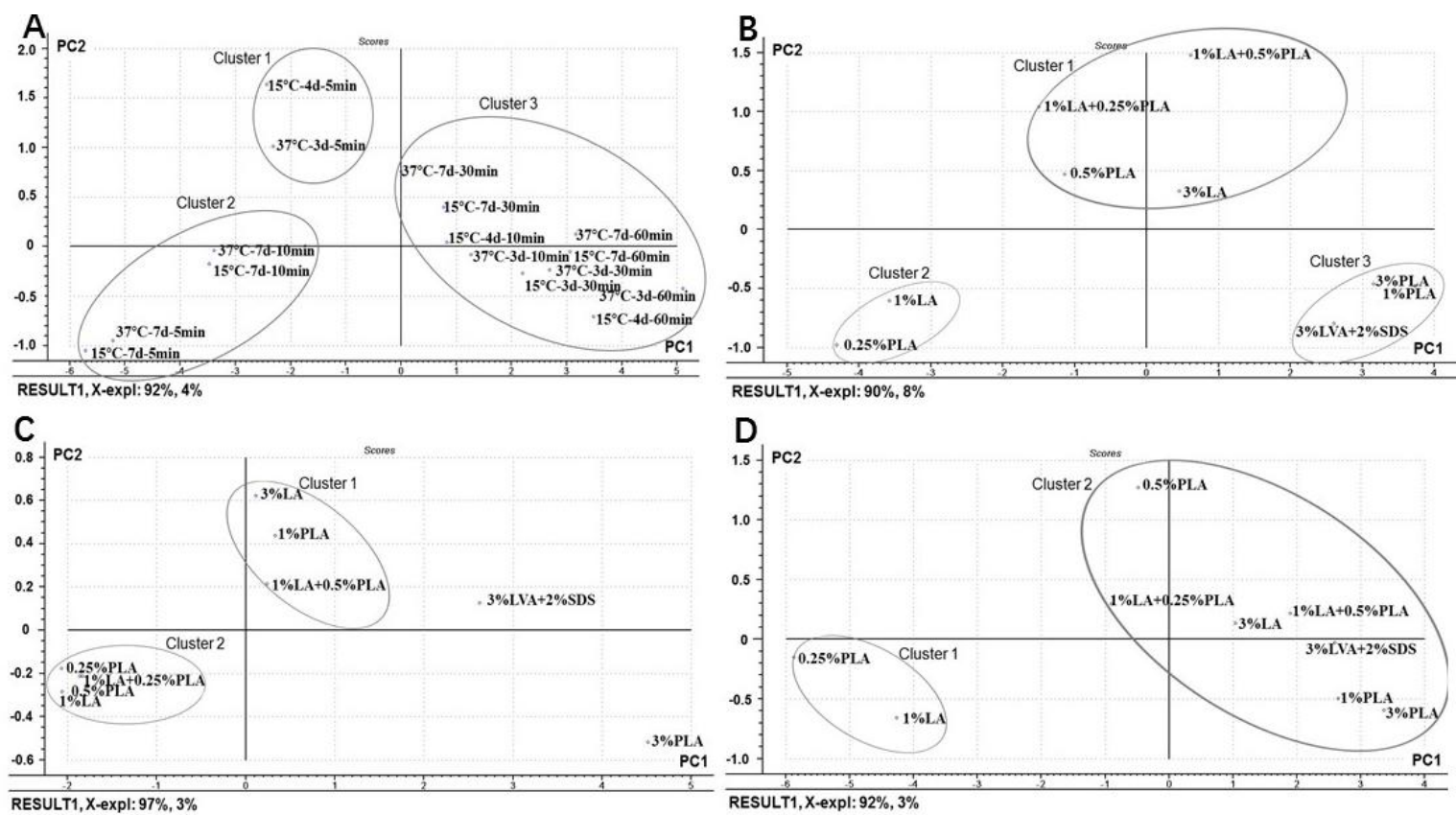

Fig. 4. 